spacecraft in 2009. Because a ripple's wavelength is related to how long ago it was created, the researchers calculated that this ripple began in 1983. They say that debris from a colliding comet crashed into particles in the ring, causing it to tilt and wobble.

Another group that included Hedman and was led by Mark Showalter at the SETI Institute in Mountain View, California, found two sets of ripples in images of Jupiter's rings taken by the Galileo and New Horizons spacecraft. The team reports that Jupiter's rings were hit by debris from two comets, the first of which struck in 1990 and the second in 1994. The authors associated this latter hit with a known Jupiter strike by the ShoemakerLevy 9 comet in July 1994.

Monitoring the rings' wobble may help astronomers to map the internal structure of the planets' cores.

Science doi:10.1126/

science.1202238; doi:10.1126/

science.1202241 (2011)

For a longer story on this research, see go.nature.com/ a5qsys

\section{CHEMISTRY}

\section{Fine-tuning polymerization}

Synthetic polymers are all around us. Researchers are working on ways to more finely control the chemical process that produces these compounds, to generate polymers with, for example, specific molecular masses and architectures.

Krzysztof Matyjaszewski at Carnegie Mellon University in Pittsburgh, Pennsylvania, and his collaborators show that applying a suitable electrochemical potential to a copper catalyst commonly used in polymerization reactions switches the catalyst between a dormant (oxidized) and an active (reduced) state. Because the polymerization reaction rate depends on the ratio of the concentrations of the two copper species, this toggling provides fine control over the yield and molecular mass of the product. Moreover, polymerization can be stopped and restarted at will by adjusting the applied potential.

The approach may be extendable to various types of polymer, and might be used to generate complex architectures, the authors say. Science 332, 81-84 (2011)

\section{EVOLUTIONARY BIOLOGY}

\section{Bad for your teeth?}

Faecal analysis has added grist to the debate over whether dietary silica contributed to the evolution of herbivores' high-crowned teeth. The compound, which is found in dust and in certain plants, such as grasses, is harder than tooth enamel and can wear teeth down.

Jürgen Hummel at the University of Bonn in Germany and his colleagues measured the levels of silica, which is not absorbed or degraded during digestion, in the faeces of 15 species of African herbivore, including giraffes, antelopes and zebras. They used this as a proxy for the amount of silica ingested, and found that herbivores with higher-crowned teeth tend to have higher levels of silica in their diet. This supports the argument that herbivores evolved these teeth to offset the dental erosion caused by their diet.

Proc. R. Soc. B doi:10.1098/ rspb.2010.1939 (2010)

\section{PALAEONTOLOGY \\ The giant rabbits of Minorca}

Fossil bones from a species of giant rabbit, Nuralagus rex, have been discovered on the Mediterranean island of Minorca, off the coast of Spain.

Meike Köhler at the Catalan Institute for Research and Advanced Studies in Barcelona, Spain, and her colleagues dated the fossils to the late Neogene period,

COMMUNITY CHOICE

The most viewed papers in science

\title{
Starved cells opt for quiescence
}

\section{HIGHLY READ \\ on jcb.rupress. org in March}

that the deprivation and addition of glucose triggers yeast cells to enter and exit from this state, respectively, regardless of which stage of the cell cycle they are in. This contradicts the common belief that cells can become quiescent only when they are in a specific phase of the cell cycle.

Isabelle Sagot and her colleagues at the National Centre for Scientific Research in Bordeaux found that Saccharomyces cerevisiae cells in all stages of the cell cycle display the cellular hallmarks of quiescence entry. The authors also found that glucose added to the cells had to be metabolized to a certain point for the cells to exit from quiescence. This suggests that quiescence signals are more closely linked to the cell's metabolic status than to the cell cycle.

J. Cell Biol. 192, 949-957 (2011)

about 5 million years ago. The outsized rabbit (reconstruction pictured) would have weighed about 12 kilograms -10 times the weight of its closest living continental relative, Oryctolagus cuniculus (pictured). The hefty animal also had a relatively small skull and sensory organs, and lacked the backbone anatomy required for hopping - traits, the authors say, that may have evolved because the rabbits had no predators on the island. J. Vertebr. Paleontol. 31, 231-240 (2011)

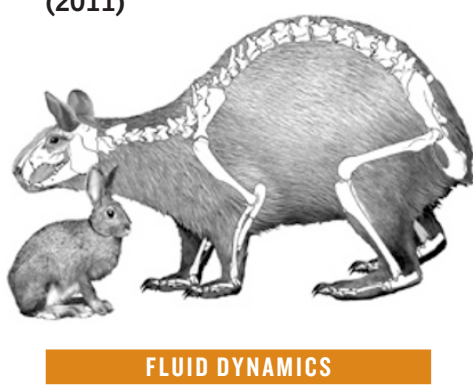

\section{Getting through a drowning machine}

People adrift in fast-moving rivers can get caught and even drown in hydraulic jumps turbulence that forms when a shallow, fast-moving waterway spills into a slower and deeper section. By creating a small hydraulic jump in the lab, researchers have determined the factors that affect how long different objects remain caught in these 'drowning machines.'

Pinaki Chakraborty and his colleagues at the University of Illinois in Urbana observed the time it took objects of different shapes and buoyancies, such as a ball and a bottle, to emerge from the jump. Using these data, they calculated that light objects remain in the rolling eddy for several minutes, whereas denser items - including humans - should be expelled after only seconds. Because object buoyancy affected residence time, the authors suggest that public-safety agencies test the best strategy for people to use if they fall into a drowning machine: remaining still, and thus more buoyant, or swimming with the current to emerge from the jump sooner. Proc. Natl Acad. Sci. USA doi:10.1073/pnas.1015183108 (2011)

\section{$\rightarrow$ NATURE.COM}

For the latest research published by Naturevisit:

www.nature.com/latestresearch 